\title{
DYNAMIC RESPONSE OF TOWER STRUCTURES
}

\author{
Liga Gaile, Ivars Radinsh ${ }^{2}$ \\ 1- Riga Technical University, Department of Structural Engineering \\ 16/20 Azenes street, Riga LV 1048, Latvia \\ Ph.: +(371)27555757, fax: +(371)67089195, e-mail: smgprojects @ inbox.lv \\ 2- Riga Technical University, Department of Structural Mechanics \\ 16/20 Azenes street, Riga LV 1048, Latvia \\ Ph.: 371+ 67089262, fax: 371+67089195, e-mail: ivarsr@bf.rtu.lv
}

\begin{abstract}
The present study focuses on the tower type structures response to the dynamic loads. The study analyzes the possible mode shapes regarding to tower structure. The estimation of mode shapes and their dependence from structural changes was made for an existing tower structure. To get an acceptable tower's vibration level and avoid possibility of resonance effect from usual serviceability loads it was evaluated options to change natural frequencies of the structure. It is performed existing 36m high sightseeing tower dynamic analysis and proposed potential solutions to increase critical natural frequencies of the structure. In this study to obtain dynamic parameters of the sightseeing tower structure have been used finite element models and calculation techniques.
\end{abstract}

Keywords: dynamic parameters, frequency, mode shapes, steel tower, vibrations.

\section{Introduction}

The environment is affected when structures are being built and this can happen in a several ways. One of the aspects is amount of materials used for the construction of structure. To save a natures recourses, energy and due to economical reasons the steel tower type structures nowadays are getting remarkably flexible, low in damping and light in weight. Traditionally for such type of structures dynamic analysis are performed and dynamic parameters such as fundamental frequencies, mode shapes and damping ratios are found to evaluate wind induced vibrations and effects on the structure. Even most advanced and comprehensive codes concentrate mainly on these issues, including the Eurocodes. In case of the steel lattice sightseeing towers with low natural frequency of the structure human and structure interaction could play a role in the tower design. Human walking induces dynamic and time varying forces. Those forces have components in vertical, lateral and longitudinal directions. The lateral forces are a consequence of the sideway oscillation of the gravity centre of a human's body while stepping alternatively with the right or left foot forwards [1]. Published data on dynamics loads quote that pedestrian vertical and longitudinal walking on stationary pavements fundamental frequency is $2.0 \mathrm{~Hz}$ for normal walk, $1.7 \mathrm{~Hz}$ for slow walk and 2.3 for fast walk. Horizontal fundamental frequency is $1.0 \mathrm{~Hz}$ for normal walk, $1.85 \mathrm{~Hz}$ for slow walk and 1.15 for fast walk [2]. In a case of the tower structure there is an interest in horizontal and longitudinal component of the pacing frequency. Recently, there has been growing tendency to construct light weight foot bridges. Due to experienced problems in some of those structures with lateral vibrations there have been performed studies about phenomenon of synchronous lateral excitation. It is noted that humans are much more sensitive to lateral vibration than vertical one. Even if horizontal vibration is only 2-3 millimeters lateral motion affects balance and pedestrians tend to walk with their feet further apart which increases the lateral force imparted by individuals. In order to maintain balance, pedestrians tend to synchronize their footsteps with the motion of the structure. This instinctive behavior ensures that dynamic forces are applied at the resonant frequency of the structure and increase the motion even more. As the motion increases also increases the 
synchronization between pedestrians. It will not go infinity but reaches a steady state by people stopping when motion becomes too uncomfortable [3]. It is presumed that the same processes will take place on the sightseeing towers. Wind forces will promote initiation of the lateral motion and because of adaptive nature of the human beings lateral vibration will have a self exited nature until some point.

\section{Study and methods}

There are several important aspects for the sightseeing towers. Firstly it should be esthetically pleasing, incorporated and consistent with the surrounding environment, comfortable stairs for the public use. In the general these aspects are governing the towers shape and structure. Unlike industrial steel towers the sightseeing towers structures will be more complicated and new aspects can rose during the designing process. The main purpose of this study is to identify whether a light weight sightseeing tower with particular steel structure is susceptible to the human induced excitation. In this paper there are analyzed possible mode shapes and corresponding fundamental frequencies of the existing steel core sightseeing tower. There is evaluated potential to change fundamental frequency of the tower by making amendments to the structure itself.

The studied steel core sightseeing tower is located in Dzintari, Jurmala city, Latvia (Fig. 1). It is open for public since $15^{\text {th }}$ of May 2010. Total height of the tower is $36.48 \mathrm{~m}$. All elements inner and outer core, platforms, and stairs are made of steel except the wooden cladding on the facades of the steel cores. The structural configuration of the tower is provided in Fig. 2. Structure consists of braced inner core with dimensions $1500 \times 1500 \mathrm{~mm}$ made from tubes with cross section 200x200x8 and outer core with dimensions 4240x4240mm made from tubes with cross section $140 \times 140 \times 5$. Outer core does not have any vertical bracing as this was requested by architectural concept. Inner and outer cores are connected only with steel stairs.

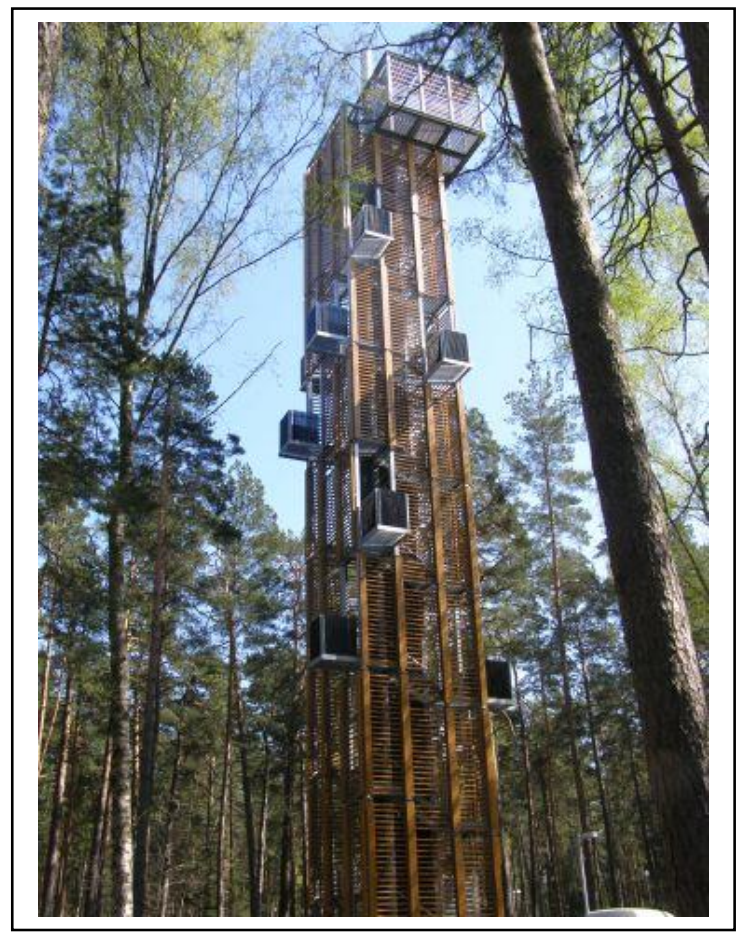

Fig.1. Sightseeing tower in Dzintari

Since the tower was opened for public there have been complaints about tower excessive vibration. The human perception of vibration is very sensitive and reaction is substantially psychological. There for it should be analyzed whether these vibrations are realistic or just perceived by the human visual stimuli. 
The literature [5] provides a recommendation whenever fundamental frequencies are close to a critical range (from point of view of the pedestrian excitation) to use a more precise numerical model, because hand formulas and simplified methods are not enough for assessment of fundamental frequencies. The finite element software is widely spread and accepted as more precise numerical model. To evaluate degree of vibration there was established fundamental frequencies and critical mode shapes of the existing tower using three dimensional finite element models created by structural analysis software STRAP 12.5. This software also was used to assess how the changes in the structure of tower affects the fundamental frequencies and mode shapes.

To determine structural system fundamental frequency and mode shapes by solving undamped free vibration equation [4]:

$$
[M]\{\ddot{u}\}+[K]\{u\}=0
$$

where $\mathrm{M}$ is the mass matrix, $\mathrm{K}$ is the stiffness matrix, $\ddot{u}$ is the acceleration vector and $\mathrm{u}$ is the displacement vector, for extraction of eigenvalues the structural analysis software uses subspace iteration technique.

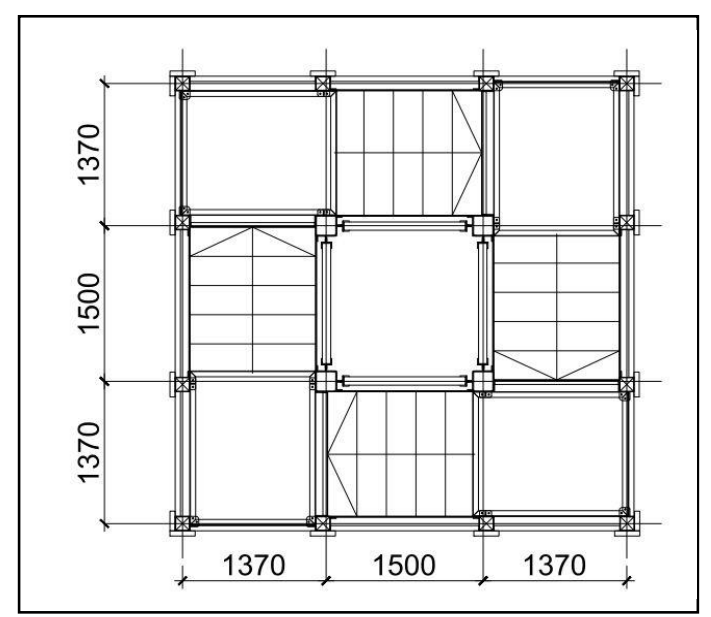

Fig.2. Plan of the Sightseeing tower in Dzintari

Tower was modeled step by step to evaluate main component influence on its fundamental frequency. The created finite element (FE) models of the tower are summarized in table 1.

Table 1.

FE models of the tower

\begin{tabular}{|c|c|c|c|}
\hline $\begin{array}{c}\text { Model } \\
\mathbf{N r} .\end{array}$ & Description & $\begin{array}{c}\text { Inner core dimensions } \\
(\mathbf{m m})\end{array}$ & $\begin{array}{c}\text { Outer core } \\
\text { dimensions }(\mathbf{m m})\end{array}$ \\
\hline 1. & Only inner core & $1500 \times 1500$ & - \\
\hline 2. & Inner and outer core & $1500 \times 1500$ & $2870 \times 2870$ \\
\hline 3. & Inner and outer core & $1500 \times 1500$ & $4240 \times 4240$ \\
\hline 4. & Inner and outer core & $1500 \times 1500$ & $5610 \times 5610$ \\
\hline 5. & Inner and outer core & $1500 \times 1500$ & $6980 \times 6980$ \\
\hline 6. & Inner and outer core, stairs & $1500 \times 1500$ & $4240 \times 4240$ \\
\hline 7. & $\begin{array}{c}\text { Inner and outer core, stairs, } \\
\text { eccentric platforms }\end{array}$ & $1500 \times 1500$ & $4240 \times 4240$ \\
\hline 8. & Only inner core & $2000 \times 2000$ & - \\
\hline 9. & Inner and outer core & $2000 \times 2000$ & $3370 \times 3370$ \\
\hline 10. & Inner and outer core & $2000 \times 2000$ & $4740 \times 4740$ \\
\hline 11. & Inner and outer core & $2000 \times 2000$ & $6110 \times 6110$ \\
\hline 12. & Inner and outer core & $2000 \times 2000$ & $7480 \times 7480$ \\
\hline
\end{tabular}


During the creation process of the FE models and their evaluation for the base model was taken the behavior of the model Nr. 6 with inner core, outer core and stairs but without eccentric platforms. Outer core of the tower behaves more as unbraced in vertical direction. On this base more simplified models were created without any bracing in outer core façades and the last tower platform created as horizontal diaphragm.

\section{Results}

For all twelve FE models fundamental natural frequencies were calculated and summarized in table 2. There were established first three critical mode shapes which are the same for all twelve FE models (Fig. 3).

Table 2.

\section{Calculated fundamental frequencies of the tower}

\begin{tabular}{|c|c|c|c|}
\hline $\begin{array}{c}\text { Model } \\
\text { Nr. }\end{array}$ & $\mathbf{1}^{\text {st }}$ mode (Hz) & $\mathbf{2}^{\text {nd }}$ mode $(\mathbf{H z})$ & $\begin{array}{c}\mathbf{3}^{\text {rd }} \text { mode }(H z) \\
\text { Torsional mode }\end{array}$ \\
\hline 1. & 1.521 & 1.521 & 6.051 \\
\hline 2. & 0.874 & 0.874 & 2.777 \\
\hline 3. & 0.874 & 0.874 & 2.031 \\
\hline 4. & 0.874 & 0.874 & 1.589 \\
\hline 5. & 0.874 & 0.874 & 1.301 \\
\hline 6. & 1.506 & 1.511 & 2.010 \\
\hline 7. & 1.279 & 1.296 & 1.914 \\
\hline 8. & 1.955 & 1.955 & 6.376 \\
\hline 9. & 1.151 & 1.151 & 3.206 \\
\hline 10. & 1.131 & 1.131 & 2.434 \\
\hline 11. & 1.111 & 1.111 & 1.917 \\
\hline 12. & 1.09 & 1.09 & 1.561 \\
\hline
\end{tabular}

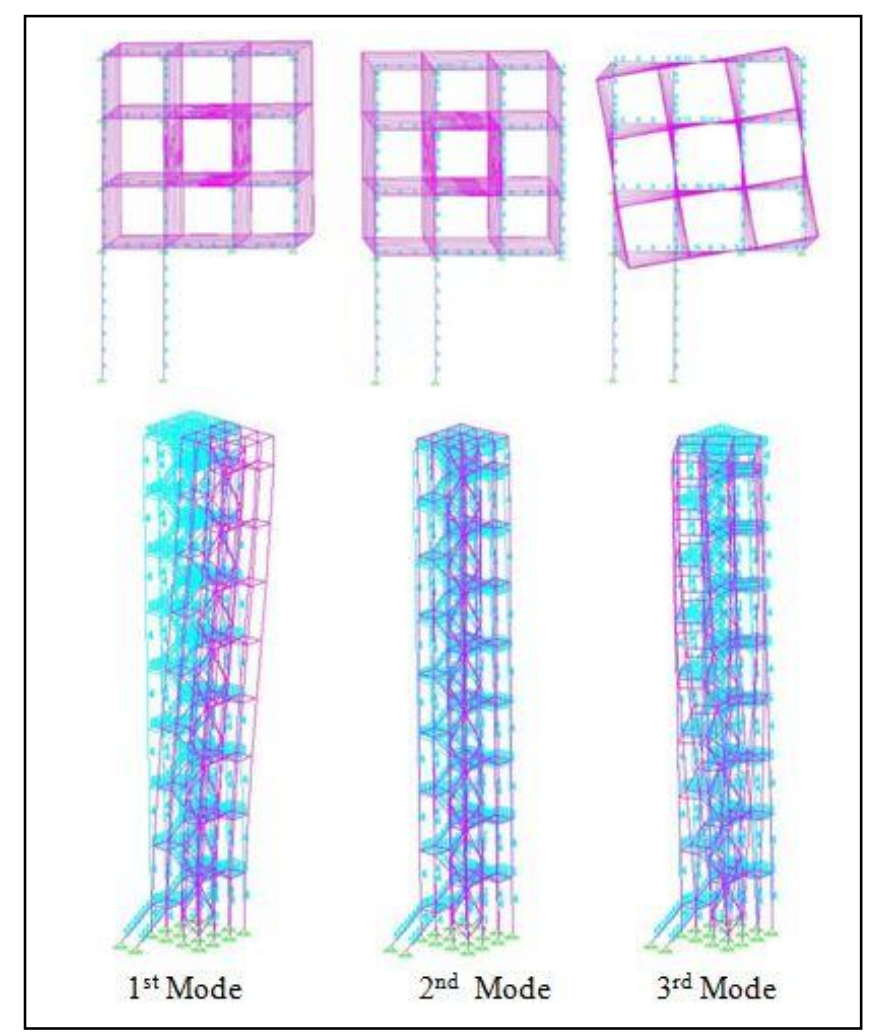

Fig.3. Mode shapes for the FE model Nr. 6 
As it was expected the first and second mode shape fundamental frequencies mainly depend on the internal core stiffness (see FE Nr. 1-5 and Nr. 8-12). Adding the outer core whose stiffness was relatively small because of the vertical bracing absence the first and second mode shape fundamental frequency basically did not change and outer core acts more like a mass which decreases frequency of the entire tower. FE model Nr. 6 and 7 results shows that stairs provides some degree of restraint for the transversal directions and increase fundamental frequencies for the first and second mode shapes comparing to the models without stairs.

By analysing these results the most changes in the fundamental frequency was notified in the torsional mode shape ( $3^{\text {rd }}$ mode shape). Adding the outer core to the structure dramatically decreased fundamental frequency and it is sensitive to the outer core placement with regards to the inner core. To demonstrate the outer core placement with regards to the inner core, both cores stiffness and mass influence on the torsional mode shape fundamental frequency it is proposed to find the equivalent square hollow box cross section with the same mass as the actual tower cross section mass and equivalent square hollow core cross section with the same torsional stiffness as the actual tower cross section torsional stiffness (see Fig. 4).

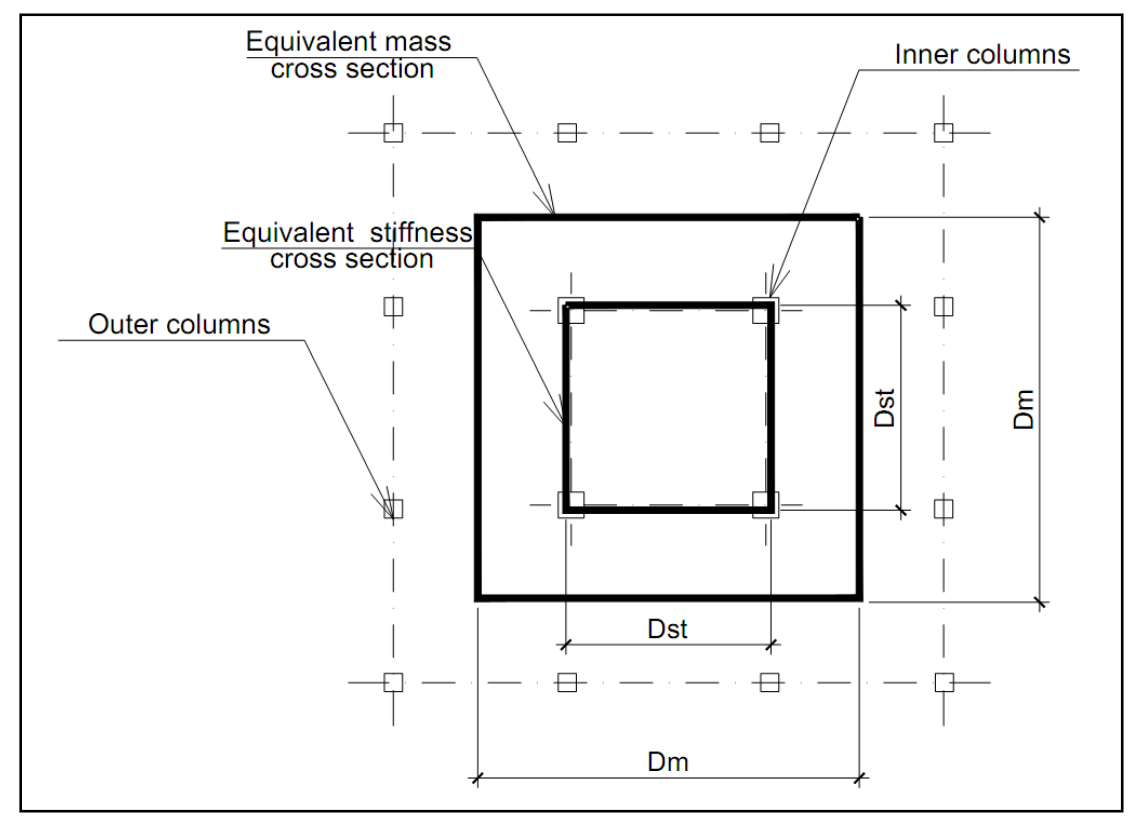

\section{Fig.4. Tower equivalent mass and stiffness cross sections}

Stiffness and mass cross section dimensions Dm and Dst coincides in case of FE models Nr.1 and Nr. 8 where tower is modeled only with one core. For the rest of the models where outer core as well as inner core was modeled, the equivalent cross section dimensions Dm and Dst do not coincides. In this particular tower case the inner core stiffness is higher because of the vertical bracing. Therefore dimension Dst is closer to the inner core width dimension. The Fig. 5 shows that if ratio Dm/Dst is growing the fundamental frequency is rapidly decreasing. Respectively when the mass around the centre of the tower is distributed further and stiffness is concentrated closer to the centre of the tower, there is a rapid decrease of the fundamental frequency for the torsional mode shape and it could lead to the critical range where tower is susceptible to the resonance effects induced by the human structure interaction.

Keeping in mind the above chart FE model Nr. 6 was amended and added vertical bracing to the outer core. Increasing outer core stiffness the ratio Dm/Dst came close to value 1.00 and torsional mode fundamental frequency rose to $6.06 \mathrm{~Hz}$. 
For the FE model Nr. 7 which is modeled to most precisely reflect the existing tower with added eccentrical platforms and cladding elements, as it was expected the fundamental frequency decreased due to additional mass.

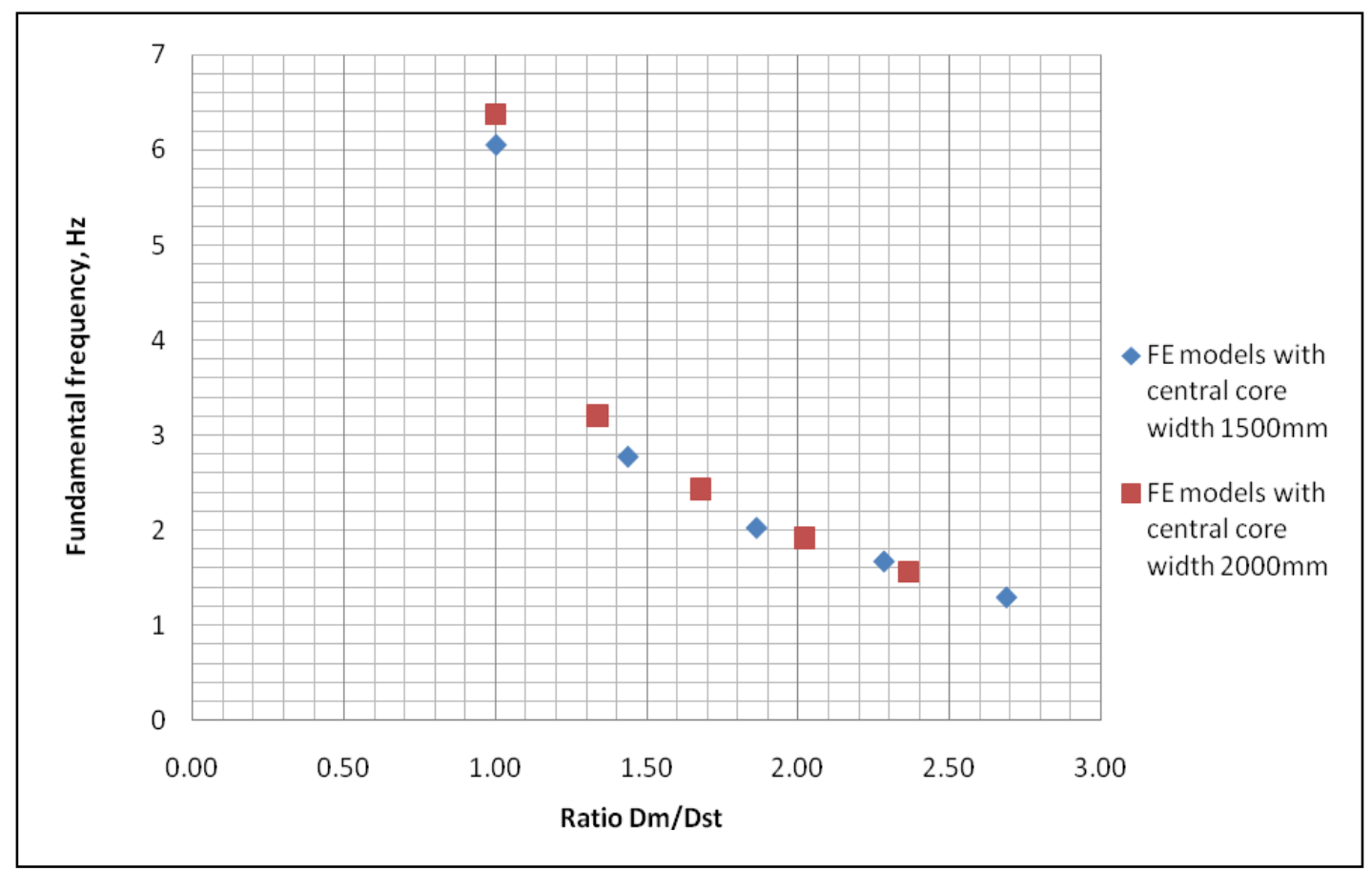

Fig.5. Fundamental frequency of the third mode shape (torsional) dependence from the ratio Dm/Dst

\section{Conclusions}

All tower models' fundamental frequencies $f$ of $1^{\text {st }}$ and $2^{\text {nd }}$ mode shapes and tower models' with two different stiffness cores fundamental frequency $f$ of $3^{\text {rd }}$ mode shape lie in an interval $1.15 \mathrm{~Hz} \leq f \leq 2.3$. The tower being within this interval makes it susceptible of excitation by harmonic of pedestrian excitation. In conclusion the existing tower should be the object for the further dynamic assessment to pedestrian movement.

The tower with two structural cores and internal core being remarkably stiffer, increase of outer core perimeter leads to the decrease of the tower fundamental frequency for $3^{\text {rd }}$ (torsional) mode shape. It is suggested to link this effect with changes of stiffness and mass ratio in the structure.

By adding additional elements around the tower's inner core (dimension 1500x1500mm) the fundamental frequency for the $1^{\text {st }}$ and $2^{\text {nd }}$ mode shape decreases by $43 \%$ but for the $3^{\text {rd }}$ mode shape it decreases by $54-78 \%$. The author considers it is because the tower stiffness and tower mass changes according to the different relationships.

By adding an additional vertical bracing to the outer core it is possible to bring fundamental frequency of 3rd mode shape out of the critical range.

\section{Summary}

The first three mode shapes susceptible of excitation by harmonic of the pedestrian movement are critical to the tall sightseeing steel towers that have more than one structural core with different degree of stiffness in respect to each other.

Published data for the tower and human interaction are thin therefore further research is required which includes load models for pedestrian streams, possible design modifications by increasing fundamental frequencies or development of low cost damping devices to reach the vibration comfort range in terms of the acceleration. 


\section{References}

1. Franck L. Synchronous Lateral Excitation of Footbridges, Swiss Federal Institute of Technology, Swiss, 2009, p.5.

2. Bachman H., Ammann W. Vibration in Structures Induced by Man and Machines. Structural Engineering Document 3e, International Association for Bridge ans Structural Engineering (IABSE), ch. 2: Man Induced Vibrations, Appendix A: Case Reports, 1987.

3. Fujino Y., Pacheco B., Nakamura S., Warnitchai P. Sychronization of human walking observed during lateral vibration of human walking observed during lateral vibration of a congested pedestrian bridge. Earthquake Engineering and Structural Dynamic (22), p. 741-758.

4. MacLeod I. A., Modern Structural analysis. Modeling Process and Guidance, 2005, p. 138-139. (in English).

5. Reaserch Fund for Coal and Steel. Human induced Vibrations of Steel Structures. Design of Footbridges. Background Document.RFS2-CT-2007-00033, 2007, p. 5-6. 